\title{
Generation Y: Problematische Auswirkungen auf die psychiatrisch-psychotherapeutischen Fächer - Pro \& Kontra
}

\author{
Generation Y: Problematic Consequences for Psychiatry and Psychotherapy - \\ Pro \& Contra
}

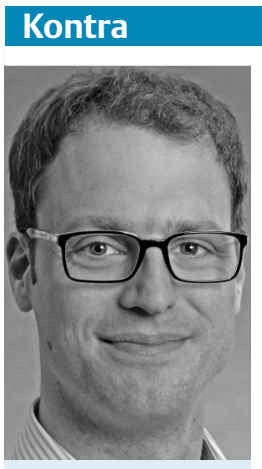

Berend Malchow
Grob gesagt bezeichnet das Konstrukt der "Generation Y“ die nach 1980 geborenen Mitglieder unserer Gesellschaft. Manche Publikationen beschränken die Zugehörigkeit auf die Geburtsjahrgänge 1982-2004 [1]. Die häufig synonym verwandten Anglizismen „Millenials“ oder „Digital Natives“ stehen für 2 Grundcharakteristika dieser Generation: Sie hat die Jahrtausendwende im Teenageralter erlebt und ist von Kindesbeinen an mit dem Internet vertraut. Außerdem sei sie emotional und finanziell sehr behütet aufgewachsen, habe viel Lob erfahren, sei sehr gut ausgebildet und gehen entsprechend selbstbewusst zu Werke. Mit diesen Attributen ausgestattet strömen die Mitglieder der „Generation Y“ nun seit einigen Jahren von den Hochschulen an ihre ersten Arbeitsplätze. Mit ihren hohen Erwartungen an Arbeitgeber und Arbeitsplatz bringen sie ihre Arbeitgeber an den Rand der Verzweiflung: Der Beruf soll spannend sein, herausfordernd, inhaltlich und gesellschaftlich wertvoll und natürlich problemlos vereinbar mit einer Familiengründung. Konkret: Er soll möglichst keine Überstunden beinhalten, sehr wohl aber die Finanzierung von Krippenplatz oder Tagesmutter für die Kinder sowie der kompletten Aus-, Fort- und Weiterbildung durch den Arbeitgeber. Bezüglich der medizinischen Disziplinen schnellen in Zeiten unbesetzter Arztstellen die Forderungen an einen potenziellen Arbeitgeber in die Höhe.

Nun wird man zweifelsohne nicht jedem Mitglied der „Generation Y“ alle obigen Attribute zuschreiben können. Trotzdem stellt diese Mitarbeitergeneration auch für psychiatrische Kliniken eine Herausforderung dar, wenn auch eine, welche gemeistert werden kann. Die Psychiatrie erfüllt nämlich bereits alle Forderungen der „Ypsiloner“. Die alltägliche Arbeit mit klinischen, wissenschaftlichen und vielleicht sogar lehrenden Tätigkeiten ist allein schon ob der mannigfaltigen und individuell ausgestalteten Krankheitsbilder extrem vielseitig und daher auch spannend und herausfordernd. Für die Krankheitsbilder existieren so viele gute und differenzierte Therapieangebote wie nie zuvor. Bereits junge Assistenzärzte und -ärztinnen kommen sowohl mit anderen medizinischen Fachgebieten wie auch anderen Disziplinen, seien es die Rechtswissenschaften oder die staatliche Exekutive, in Kontakt. Seelische Erkrankungen und deren Manifestationen berühren ebenso Gebiete der Philosophie, der Soziologie sowie der Künste. Wissenschaftlich gesehen boomen die Neurowissenschaften ungebremst, ohne dass ein Ende in Sicht wäre, und fundierte Kenntnisse über die normale und gestörte Funktionsweise des Gehirns nehmen exponentiell zu.

Diese Fülle an Wissen lässt sich nur noch mit digitalen Mitteln adäquat verwalten und weiterverarbeiten, mit welchen die „Generation Y“ spätestens seit ihrer Schulzeit vertraut ist: Computer, Netzwerke, virtueller Speicher, Chats. Mit Kollegen konferiert man nicht am Telefon, sondern kommuniziert vorzugsweise per Internet; zu bestimmten Fragestellungen kann man sich in online-Foren austauschen, Anleitungen zu speziellen Techniken werden als Wikis ins Netz gestellt. Gleichzeitig gehen die „Ypsiloner“ durchaus kritisch mit den Informationen im Internet um und akzeptieren diese nicht fraglos. Hier müssen Vorgesetzte umdenken: Unter Umgehung der Oberärzte werden sie künftig direkt elektronisch von Assistenzärzten kontaktiert und sie müssen damit rechnen, dass sog. „Expertenwissen“ kritisch geprüft wird. Insgesamt könnte dies über die Jahre vielleicht sogar zu einem besseren Umgang mit Fehlern in der Medizin führen und das Bild des makellos weißen Kittels ablösen.
Flache Hierarchien sollten in der Psychiatrie und Psychotherapie aufgrund des jahrelangen Handelns in Teams eigentlich üblich sein; wenn nicht, sollten sie schnellstmöglich realisiert werden. Doch auch die jungen Assistenzärzte müssen sich in einer flachen Hierarchie an leichte Höhenunterschiede aufgrund unterschiedlicher Erfahrungswerte und eine gesunde Kritikfähigkeit gewöhnen. Für beide Seiten, Vorgesetzte wie auch den Nachwuchs, gilt es also dazuzulernen.

In Fragen der Arbeitszeit und der entsprechenden Freizeit hat sich in der Psychiatrie und Psychotherapie viel getan - allerdings wohl noch nicht genug, wie erste Ergebnisse einer jüngsten DGPPN-Umfrage zeigten, die auf dem DGPPN-Kongress 2012 im Rahmen des Nachwuchsprogramms lebhaft diskutiert wurden. Vielfach geforderte flexiblere Arbeitszeitmodelle insbesondere für junge Mütter und Väter, die ihren Nachwuchs auch aufwachsen sehen wollen, lassen sich gerade in der Psychiatrie gut umsetzen [2]. Denn hat sich ein Mitglied der „Generation Y“ einmal aus guten Gründen für einen Arbeitsplatz und -ort entschieden, bleibt es diesem vielfach treu. Das Ausland wurde bereits zu Schulzeiten und spätestens im Studium erobert, Kontakte pflegt man über das Internet. Auch ländliche Regionen könnten aufgrund ihrer Natur und Möglichkeiten zur gesunden und wertvollen Freizeitgestaltung für junge Familien in Zukunft wieder attraktiver werden.

Generell wichtig erscheint vor allem eines: dass nämlich Vorgesetzte bereits bei Einstellungsgesprächen obige Themen explizit ansprechen. Wenn Mitglieder der „Generation Y“ wissenschaftlich aktiv sein und an einem spannenden und herausfordernden Projekt mitarbeiten wollen und Aufgaben und eventuelle Früchte dieser Tätigkeit nicht nebulös bleiben, dann dürften die betreffenden „Ypsiloner“ - insbesondere gegen Projektende - auch unbezahlte Überstunden und Forschung in der Freizeit klaglos in Kauf nehmen. Zudem hilft Transparenz in allen Bereichen 
auch Arbeitgebern und saturierten Kollegen, ihr eigenes Tun und Handeln zu hinterfragen - was manche sicher als unbequem empfinden. Aber waren diese Kollegen nicht selbst einmal jung und haben ihrerseits einen Umgang auf gleicher Augenhöhe, wenn auch manchmal nur im stillen Kämmerlein, herbeigesehnt und gefordert? Die Generation Y fordert also schlicht und einfach, was eigentlich Normalität sein sollte. Eine weitere Voraussetzung sind natürlich auch entsprechende finanzielle Rahmenbedingungen seitens Politik und Krankenkassen. Vielleicht bietet das neue Entgeltsystem in dieser Hinsicht eine Chance. Sollte sich das Gegenteil abzeichnen, wird unsere psychi- atrisch und psychotherapeutisch ausgebildete „Generation Y“ jedenfalls ein würdiger Diskussionspartner sein.

Eine Karriere in der Psychiatrie und Psychotherapie ist im Grunde wie maßgeschneidert für die „Generation Y“ - jetzt müssen wir diese Erkenntnis nur noch generationsspezifisch kommunizieren. Schließlich twittert inzwischen selbst der Papst!

\section{Literatur}

1 Howe N, Strauss W. Millennials Rising: The Next Generation. New York: Vintage; 2003

2 Schneider F, Falkai P, Maier W. Psychiatrie 2020 plus - Perspektiven, Chancen und Herausforderungen. 2. aktualisierte Auflage. Berlin: Springer; 2012
Korrespondenzadresse

\section{Berend Malchow}

Klinik für Psychiatrie und Psychotherapie, Universitätsmedizin Göttingen Von-Siebold-Straße 5

37075 Göttingen

Berend.Malchow@medizin.uni-goettingen.de

Bibliografie

DOI http://dx.doi.org/

10.1055/s-0032-1332830

Psychiat Prax 2013; 40: 63-64

(c) Georg Thieme Verlag KG

Stuttgart · New York

ISSN 0303-4259 